\title{
The Gravitational Field in the Relativistic Uniform Model within the Framework of the Covariant Theory of Gravitation
}

\author{
Sergey G. Fedosin \\ PO box 614088, Sviazeva str. 22-79, Perm, Perm Krai, Russia \\ fedosin@hotmail.com
}

Keywords: Relativistic uniform system; covariant theory of gravitation; gravitational field; energy.

\begin{abstract}
For the relativistic uniform system with an invariant mass density the exact expressions are determined for the potentials and strengths of the gravitational field, the energy of particles and fields. It is shown that, as in the classical case for bodies with a constant mass density, in the system with a zero vector potential of the gravitational field, the energy of the particles, associated with the scalar field potential, is twice as large in the absolute value as the energy defined by the tensor invariant of the gravitational field. The problem of inaccuracy of the use of the field's stress-energy tensors for calculating the system's mass and energy is considered. The found expressions for the gravitational field strengths inside and outside the system allow us to explain the occurrence of the large-scale structure of the observable Universe, and also to relate the energy density of gravitons in the vacuum field with the limiting mass density inside the proton. Both the Universe and the proton turn out to be relativistic uniform systems with the maximum possible parameters. The described approach allows us to calculate the maximum possible Lorentz factor of the matter particles at the center of the neutron star and at the center of the proton, and also to estimate the radius of action of the strong and ordinary gravitation in cosmological space.
\end{abstract}

\section{Introduction}

The relativistic theory describes the physics of phenomena more precisely than classical mechanics. This leads to the fact that an ideal uniform model of a body with a constant mass density must be replaced by the relativistic uniform model. In the relativistic model the mass density can be the coordinate function, but it is considered a constant invariant mass density in the reference frames, associated with the particles that make up the body. Due to the motion of the particles the effective mass density in the system differs from the invariant values, which introduces additional corrections into the values of the field functions and into the system's energy.

Various properties of the relativistic uniform system were discussed earlier in [1-2]. The purpose of this paper is to verify the relation between the energy of the particles in the scalar gravitational potential and the proper energy of the gravitational field, in the framework of the covariant theory of gravitation and relativistic uniform model. We will use the covariant theory of gravitation instead of the generally accepted general theory of relativity. This is due to the fact that in the general theory of relativity there is no stress-energy tensor of the gravitational field, which does not allow to uniquely calculate the energy density and the energy flux of the field inside or outside the body [3,4]. On the other hand, with the help of the covariant theory of gravitation it is possible to construct the models of stars and to estimate their parameters [5], and to refine the virial theorem [6]. Besides in the framework of the covariant theory of gravitation the gravitational field was included into the general field as a separate component [7]. The metric near the solitary massive body was determined in [8], with the help of which it was shown in [9] that the covariant theory of gravitation successfully explains the anomalous precession of Mercury's perihelion, the deviation of particles and light in the gravitational field, the gravitational time delay and the gravitational redshift of light, as well as the Pioneer anomaly [10].

Another characteristic feature of the covariant theory of gravitation is the presence of the four-potential of the gravitational field, which contains the vector potential in addition to the scalar potential of the field. With the help of the vector potential, the supplementary additives to the 
gravitational field strengths and to the gravitational torsion field are formed, which in gravitoelectromagnetism, as a special case of the general theory of relativity, are called the gravitoelectric field strengths and gravitomagnetic field strengths, respectively. These additives are required to take into account the delay in the propagation of the gravitational interaction, just as it occurs in electromagnetism with the propagation of the electromagnetic interaction. Due to the vector potential and the torsion field various effects are possible, including gravitational induction $[11,12]$.

The covariant theory of gravitation also differs from modified versions of the general theory of relativity, such as $f(R), f(T), f(R, T)$ models or the Einstein's model with the cosmological constant $\Lambda$ (Einstein- $\Lambda$ model). The common purpose of these models is their application in cosmology, where they allow taking into account the effects, which are now attributed to the dark energy and dark matter. But in a number of works, these gravitation models are also applied to a system of limited size. For example, in [13] the Palatini $f(R)$ gravity is used to describe dynamical instability in a collapsing self-gravitating spherical system of stellar sizes, and in [14] $f(R, T)$ gravity is applied to a self-gravitating anisotropic cylindrical system, which can contain an internal cavity as a result of explosive expansion. In [15], the field equations and dynamic equations for the matter of a compact star are studied using $\alpha R^{2}+\beta\left(R_{\gamma \delta} T^{\gamma \delta}\right)$ gravity, and in [16] $f(T)$ gravity is used to simulate the equation of the state of the relativistic matter. Interpretation of the results, as always, is most clear in the weak field approximation, while Newtonian and post-Newtonian approximations usually serve as the starting point.

In contrast to these modified theories of gravitation, in the weak field limit the covariant theory of gravitation turns into the Lorentz-invariant theory of gravitation and does not contain any elements associated with the dark energy and dark matter. However, the application of the covariant theory of gravitation to the relativistic uniform system leads to specific features for the potentials and the gravitational field strengths. As will be shown later, these features are of greatest importance for the objects, in which the product of the object's radius by the square root of the mass density reaches its maximum. In particular, we will apply the expressions for the gravitational field strengths to the observable Universe, to the neutron star and to the proton, which will allow us to explain some properties of these objects.

\section{The Field Functions}

As a uniform relativistic system the spherical system is considered, which consists of the particles that can also have the electrical charge. The stability of the system is maintained by the action of its proper gravitation, the internal pressure field and the acceleration field of the particles $[17,18]$. The field functions are calculated on the assumption that there is no general rotation of the particles in the system, they move randomly and therefore the total vector field potentials on the average tend to zero.

The equation for the gravitational scalar potential inside the sphere and its solution in the special relativity limit have the following form [2]:

$$
\begin{gathered}
\Delta \psi_{i}=4 \pi G \rho_{0} \gamma^{\prime} \\
\psi_{i}=\frac{G c^{2} \gamma_{c}}{\eta} \cos \left(\frac{a}{c} \sqrt{4 \pi \eta \rho_{0}}\right)-\frac{G c^{3} \gamma_{c}}{r \eta \sqrt{4 \pi \eta \rho_{0}}} \sin \left(\frac{r}{c} \sqrt{4 \pi \eta \rho_{0}}\right) \approx \frac{2 \pi G \rho_{0} \gamma_{c}\left(r^{2}-3 a^{2}\right)}{3} .
\end{gathered}
$$

In (1) the Lorentz factor of the particles is $\gamma^{\prime}=\frac{1}{\sqrt{1-v^{\prime 2} / c^{2}}}, \mathbf{v}^{\prime}$ is the average velocity of an arbitrary particle inside the sphere, $\gamma_{c}$ is the Lorentz factor of the particles at the center of the sphere, $c$ is the speed of light, $\eta$ is the acceleration field coefficient, $G$ is the gravitational constant, $\rho_{0}$ is the mass density of the particle in the reference frame associated with the particle, $a$ 
is the radius of the sphere, the index $i$ differentiates the internal gravitational scalar potential $\psi_{i}$ from external potential $\psi_{o}$, which is generated by the sphere outside its limits with $r \geq a$. Both potential $\psi_{i}$ and $\gamma^{\prime}$ are the functions of the current radius $r \leq a$ inside the sphere and do not depend on the angular variables.

The dependence of $\gamma^{\prime}$ on the radius was found in [1]:

$$
\gamma^{\prime}=\frac{c \gamma_{c}}{r \sqrt{4 \pi \eta \rho_{0}}} \sin \left(\frac{r}{c} \sqrt{4 \pi \eta \rho_{0}}\right) \approx \gamma_{c}-\frac{2 \pi \eta \rho_{0} r^{2} \gamma_{c}}{3 c^{2}} .
$$

It is precisely because of the presence of a sine in the expression for the Lorentz factor in (2), that the periodic functions also appear in the solution of (1) for $\psi_{i}$. The approximate expressions on the right-hand side of (1) and (2) appear if we take the first expansion terms of the periodic functions with regard to the smallness of their argument. As we can see, the expression for $\psi_{i}$ only in the first approximation coincides with the classical expression for the scalar potential inside the uniform sphere in the right-hand side of (1), which is a consequence of the difference between the relativistic and classical uniform systems.

For the external gravitational potential $\psi_{o}$ of the fixed sphere, filled with moving particles, we obtain the following:

$$
\psi_{o}=-\frac{G c^{3} \gamma_{c}}{r \eta \sqrt{4 \pi \eta \rho_{0}}}\left[\sin \left(\frac{a}{c} \sqrt{4 \pi \eta \rho_{0}}\right)-\frac{a}{c} \sqrt{4 \pi \eta \rho_{0}} \cos \left(\frac{a}{c} \sqrt{4 \pi \eta \rho_{0}}\right)\right] .
$$

As the current radius $r$ increases, this potential tends to zero. In addition, on the surface of the sphere at $r=a$ the external gravitational potential $\psi_{o}$ in (3) coincides exactly with the internal gravitational potential $\psi_{i}$ in (1).

The mass $m$ is defined as the product of the mass density $\rho_{0}$ by the sphere's volume $V_{s}$. However, the actual gravitational field outside the sphere is defined by the mass $m_{b}$, which according to [1] is equal to:

$$
\begin{aligned}
& m_{b}=\rho_{0} \int \gamma^{\prime} d V_{s}=\frac{c^{3} \gamma_{c}}{\eta \sqrt{4 \pi \eta \rho_{0}}}\left[\sin \left(\frac{a}{c} \sqrt{4 \pi \eta \rho_{0}}\right)-\frac{a}{c} \sqrt{4 \pi \eta \rho_{0}} \cos \left(\frac{a}{c} \sqrt{4 \pi \eta \rho_{0}}\right)\right] \approx \\
& \approx m \gamma_{c}\left(1-\frac{3 \eta m}{10 a c^{2}}\right) .
\end{aligned}
$$

The mass $m_{b}$ represents the sum of the invariant masses of all the particles in the system, which is equal to the gravitational mass of the system $m_{g}$. In view of the definition of the mass $m_{b}$, from (3) it follows:

$$
\psi_{o}=-\frac{G m_{b}}{r} \approx-\frac{G m \gamma_{c}}{r}\left(1-\frac{3 \eta m}{10 a c^{2}}\right) .
$$

Since after averaging over a sufficient number of particles, the internal vector gravitational potential $\mathbf{D}_{i}$ and the external vector gravitational potential $\mathbf{D}_{o}$ of the system are equal to zero because of the chaotic motion of the set of particles, the acting gravitational field strength $\boldsymbol{\Gamma}_{i}$ inside the system and strength $\boldsymbol{\Gamma}_{o}$ outside the system are actually defined only by the gradient of the corresponding scalar potential. In view of (1) and (3), for the strengths we obtain the following [1]: 


$$
\begin{aligned}
& \boldsymbol{\Gamma}_{i}=-\nabla \psi_{i}-\frac{\partial \mathbf{D}_{i}}{\partial t}=-\frac{G c^{3} \gamma_{c} \mathbf{r}}{r^{3} \eta \sqrt{4 \pi \eta \rho_{0}}}\left[\sin \left(\frac{r}{c} \sqrt{4 \pi \eta \rho_{0}}\right)-\frac{r}{c} \sqrt{4 \pi \eta \rho_{0}} \cos \left(\frac{r}{c} \sqrt{4 \pi \eta \rho_{0}}\right)\right] \approx \\
& \approx-\frac{4 \pi G \rho_{0} \gamma_{c} \mathbf{r}}{3}\left(1-\frac{4 \pi \eta \rho_{0} r^{2}}{10 c^{2}}\right) . \\
& \boldsymbol{\Gamma}_{o}=-\nabla \psi_{o}-\frac{\partial \mathbf{D}_{o}}{\partial t}=-\frac{G c^{3} \gamma_{c} \mathbf{r}}{r^{3} \eta \sqrt{4 \pi \eta \rho_{0}}}\left[\sin \left(\frac{a}{c} \sqrt{4 \pi \eta \rho_{0}}\right)-\frac{a}{c} \sqrt{4 \pi \eta \rho_{0}} \cos \left(\frac{a}{c} \sqrt{4 \pi \eta \rho_{0}}\right)\right]= \\
& =-\frac{G m_{b} \mathbf{r}}{r^{3}} \approx-\frac{G m \gamma_{c} \mathbf{r}}{r^{3}}\left(1-\frac{3 \eta m}{10 a c^{2}}\right) .
\end{aligned}
$$

The torsion field, which has the same meaning in the covariant theory of gravitation as the gravitomagnetic field in gravitoelectromagnetism, and similar in meaning to the magnetic field in electromagnetism, on the average is equal to zero, both inside and outside of the system under consideration:

$$
\mathbf{\Omega}_{i}=\nabla \times \mathbf{D}_{i}=0, \quad \mathbf{\Omega}_{o}=\nabla \times \mathbf{D}_{o}=0 .
$$

The gravitational field strength $\boldsymbol{\Gamma}_{i}$ and the torsion field $\boldsymbol{\Omega}_{i}$ together constitute the gravitational field tensor $\Phi_{\mu v}$ inside the system, and the quantities $\boldsymbol{\Gamma}_{o}$ and $\boldsymbol{\Omega}_{o}$ constitute the same tensor outside the system. Since all the expressions in the covariant theory of gravitation are derived from the principle of least action in the same way as it is done for the electromagnetic field in the curved spacetime, the gravitational field tensor is similar in its form to the electromagnetic field tensor [19]. Accordingly, the gravitational field is considered as a vector field, and the covariant theory of gravitation represents a vector-tensor theory with regard to the metric and the cosmological constant.

\section{The Energy of the Particles in the Field and the Energy of the Field itself}

In [18] the Hamiltonian of the system of particles was found taking into account the four fields acting in the system, including the acceleration field, the gravitational field, the electromagnetic field and the pressure field, and it was shown that for a stationary system in the curved spacetime the Hamiltonian is exactly equal to the relativistic energy of the system. For energy the following was found:

$$
\begin{aligned}
& E=\frac{1}{c} \int\left(\rho_{0} \vartheta+\rho_{0} \psi+\rho_{0 q} \varphi+\rho_{0} \wp\right) u^{0} \sqrt{-g} d x^{1} d x^{2} d x^{3}- \\
& -\int\left(\begin{array}{l}
\frac{c^{2}}{16 \pi G} \Phi_{\mu \nu} \Phi^{\mu \nu}-\frac{1}{4 \mu_{0}} F_{\mu \nu} F^{\mu \nu}- \\
-\frac{c^{2}}{16 \pi \eta} u_{\mu \nu} u^{\mu \nu}-\frac{c^{2}}{16 \pi \sigma} f_{\mu \nu} f^{\mu \nu}
\end{array}\right) \sqrt{-g} d x^{1} d x^{2} d x^{3} .
\end{aligned}
$$

where $\vartheta, \psi, \varphi, \wp$ are the scalar potentials of the acceleration field, gravitational field, electric field and pressure field, respectively; $\rho_{0 q}$ is the charge density of a particle in the reference frame associated with the particle; $u^{0}$ is the time component of the four-velocity of an arbitrary particle; $\Phi_{\mu \nu}$ is the gravitational tensor; $\mu_{0}$ is the magnetic constant; $F_{\mu \nu}$ is the electromagnetic tensor; $u_{\mu \nu}$ is the acceleration tensor; $\sigma$ is the coefficient of the pressure field; $f_{\mu v}$ is the pressure field tensor; $\sqrt{-g} d x^{1} d x^{2} d x^{3}$ is the moving invariant three-volume expressed in terms of the product $d x^{1} d x^{2} d x^{3}$ 
of the differentials of the space coordinates, and in terms of the square root $\sqrt{-g}$ of the determinant $g$ of the metric tensor taken with a negative sign.

In the expression for the energy (9) the first integral is related to the particles' energies in the fields' scalar potentials, and the second integral contains four tensor invariants, each of which reflects the energy of the respective field.

We will calculate in the limit of the special theory of relativity the energy of the particles in the gravitational field of the system, in which the gravitational vector potential and the gravitational torsion field on the average are equal to zero. In this case $u^{0}=c \gamma^{\prime}$ and the energy of the particles is defined as the volume integral taken of the product of the effective mass density inside the sphere $\rho=\rho_{0} \gamma^{\prime}$ by the internal scalar potential $\psi_{i}$. In view of (1-2) we obtain the following:

$$
\begin{aligned}
& \int \rho_{0} \psi_{i} \gamma^{\prime} d V=\frac{G c^{4} \gamma_{c}^{2}}{\eta^{2}}\left[\frac{c}{\sqrt{4 \pi \eta \rho_{0}}} \sin \left(\frac{a}{c} \sqrt{4 \pi \eta \rho_{0}}\right)-a \cos \left(\frac{a}{c} \sqrt{4 \pi \eta \rho_{0}}\right)\right] \cos \left(\frac{a}{c} \sqrt{4 \pi \eta \rho_{0}}\right)- \\
& -\frac{G c^{4} \gamma_{c}^{2}}{\eta^{2}}\left\{\frac{a}{2}-\frac{c}{4 \sqrt{4 \pi \eta \rho_{0}}} \sin \left(\frac{2 a}{c} \sqrt{4 \pi \eta \rho_{0}}\right)\right\} \approx-\frac{6 G m^{2} \gamma_{c}^{2}}{5 a}\left(1-\frac{4 \eta m}{7 a c^{2}}\right) .
\end{aligned}
$$

We will now calculate the volume integral taken of the tensor invariant of the gravitational field, separately for the field inside and outside the sphere. The integral of the tensor invariant is expressed in terms of the gravitational field strength $\boldsymbol{\Gamma}$ and the torsion field $\boldsymbol{\Omega}$ entering into the gravitational tensor $\Phi_{\mu \nu}$ :

$$
-\int \frac{c^{2}}{16 \pi G} \Phi_{\mu \nu} \Phi^{\mu v} d V=\frac{1}{8 \pi G} \int\left(\Gamma^{2}-c^{2} \Omega^{2}\right) d V
$$

This integral part is included in this form in the energy of the system (9) and defines there the contribution of the gravitational field. Substituting here (6-8), we find:

$$
\begin{aligned}
& -\int_{r=0}^{a} \frac{c^{2}}{16 \pi G} \Phi_{\mu v} \Phi^{\mu v} d V= \\
& =\frac{G c^{4} \gamma_{c}^{2}}{2 \eta^{2}}\left[\frac{a}{2}+\frac{c}{4 \sqrt{4 \pi \eta \rho_{0}}} \sin \left(\frac{2 a}{c} \sqrt{4 \pi \eta \rho_{0}}\right)-\frac{c^{2}}{4 \pi \eta \rho_{0} a} \sin ^{2}\left(\frac{a}{c} \sqrt{4 \pi \eta \rho_{0}}\right)\right] \approx \\
& \approx \frac{G m^{2} \gamma_{c}^{2}}{10 a}\left(1-\frac{3 \eta m}{7 a c^{2}}\right) . \\
& \quad-\int_{r=a}^{\infty} \frac{c^{2}}{16 \pi G} \Phi_{\mu v} \Phi^{\mu v} d V= \\
& =\frac{G c^{4} \gamma_{c}^{2}}{2 \eta^{2} a}\left[\frac{c}{\sqrt{4 \pi \eta \rho_{0}}} \sin \left(\frac{a}{c} \sqrt{4 \pi \eta \rho_{0}}\right)-a \cos \left(\frac{a}{c} \sqrt{4 \pi \eta \rho_{0}}\right)\right]^{2} \approx \\
& \approx \frac{G m^{2} \gamma_{c}^{2}}{2 a}\left(1-\frac{3 \eta m}{5 a c^{2}}\right) .
\end{aligned}
$$

We can sum up the integrals in (12), that is, calculate the integral of the tensor invariant over the entire volume, occupied by the field, and then compare the result with (10). We obtain the following: 
$-\frac{c^{2}}{16 \pi G} \int_{r=0}^{a} \frac{c^{2}}{16 \pi G} \Phi_{\mu \nu} \Phi^{\mu v} d V-\frac{c^{2}}{16 \pi G} \int_{r=a}^{\infty} \Phi_{\mu \nu} \Phi^{\mu v} d V=-\frac{c^{2}}{16 \pi G} \int_{r=0}^{\infty} \Phi_{\mu \nu} \Phi^{\mu v} d V=-\frac{1}{2} \int \rho_{0} \psi_{i} \gamma^{\prime} d V$.

According to (13), the integral of the tensor invariant is two times less in the absolute value than the energy of the particles in the gravitational potential.

\section{The Stress-Energy Tensor}

If we substitute (13) into the energy (9), we will see that the gravitational field energy in the form of the tensor invariant is subtracted from the energy of particles in the gravitational potential, so that the total gravitational energy of the system becomes equal to half of the energy of particles in the gravitational potential, and in this form it makes contribution into the relativistic energy of the system. The same is true for the electric field of the system under consideration, if we proceed from the similarity of the equations of both fields. Indeed, the electrostatic energy is also calculated as half of the energy of particles in the electric potential.

The total system's gravitational energy for the system under consideration can also be estimated with the help of the stress-energy tensor of the gravitational field, determined by the formula [9], [12], [19]:

$$
U^{\alpha \beta}=\frac{c^{2}}{4 \pi G}\left(g^{\alpha \nu} \Phi_{\kappa \nu} \Phi^{\kappa \beta}-\frac{1}{4} g^{\alpha \beta} \Phi_{\mu \nu} \Phi^{\mu \nu}\right),
$$

where $g^{\alpha \beta}$ is the metric tensor.

In the limit of the special theory of relativity, the time component of the given tensor is equal to:

$$
U^{00}=-\frac{1}{8 \pi G}\left(\Gamma^{2}+c^{2} \Omega^{2}\right)
$$

If we take into account that in the system under consideration the torsion field $\boldsymbol{\Omega}$ is assumed to be equal to zero, then the volume integral of $U^{00}$ will differ only by the sign from the integral of the tensor invariant in (11). Therefore, in view of (13) the total gravitational energy of the system can be expressed as follows:

$$
U=\frac{1}{2} \int \rho_{0} \psi_{i} \gamma^{\prime} d V=\frac{c^{2}}{16 \pi G} \int_{r=0}^{\infty} \Phi_{\mu \nu} \Phi^{\mu v} d V=\int_{r=0}^{\infty} U^{00} d V .
$$

Similarly, the electrostatic energy $W$ in the absence of the magnetic field $\mathbf{B}$ can be calculated by volume integration of the time component $W^{00}$ of the stress-energy tensor of the electromagnetic field:

$$
W=\frac{1}{2} \int \rho_{0 q} \varphi_{i} \gamma^{\prime} d V=-\frac{1}{4 \mu_{0}} \int_{r=0}^{\infty} F_{\mu \nu} F^{\mu v} d V=\int_{r=0}^{\infty} W^{00} d V .
$$

Equations (14) and (15) get violated as soon as the torsion field $\boldsymbol{\Omega}$ and, consequently, the magnetic field $\mathbf{B}$ appear in the system, for example, due to the coordinated motion of particles or charges.

We note that neither the stress-energy tensor of the gravitational field $U^{\alpha \beta}$ nor the stressenergy tensor of the electromagnetic field $W^{\alpha \beta}$ are part of expression (9). Therefore, in the general case, the use of the stress-energy tensors to calculate any part of the energy or the total system's energy is inappropriate. Instead of these tensors, the relativistic energy of the system (9) includes the integrals of the fields' tensor invariants. In this case, expression (9) is derived in a covariant way 
as the Hamiltonian from the principle of least action, and each field is taken into account in the Lagrangian and the energy independently of the other fields.

\section{Gravitational Field of the Universe}

In case of small sizes of $a$ and $r$ and the low mass density $\rho_{0}$ in the expressions for the potentials and the gravitational field strengths, the approximate expressions on the right-hand side of (1), (5), (6), (7) are valid. However, if $a$ or $\rho_{0}$ are large enough, it is necessary to use the exact formulas containing sines and cosines. Let us take, for example, the gravitational field strength $\boldsymbol{\Gamma}_{o}$ outside the body in (7), and let us denote: $\delta=\frac{a}{c} \sqrt{4 \pi \eta \rho_{0}}$. Then the square brackets in (7) will be written as the quantity $A=\sin \delta-\delta \cos \delta$, so that $\boldsymbol{\Gamma}_{o}$ will vary proportionally to $A$.

As long as the angle $\delta$ is small, we can approximately rely on the right-hand side of (7). If the angle $\delta$ is increased, then the quantity $A$ would first increase, and then would begin to decrease and even change its sign. So, at $\delta=\frac{\pi}{2}$ we will have $A=1$, at $\delta=\pi$ we will have $A=\pi$, and at $\delta=\frac{3 \pi}{2}$ we will have $A=-1$. If we solve the equation $\sin \delta-\delta \cos \delta=0$, we can find the angle $\delta$, at which the quantity $A$ vanishes. This angle is approximately equal to $\delta_{0} \approx 4.494$ radians.

At $\delta>\delta_{0}$ the gravitational field strength $\boldsymbol{\Gamma}_{o}$ outside the body in (7) changes its sign, so that instead of the gravitational attraction the repulsion appears. We assume that the condition $\delta>\delta_{0}$ leads to a physically unjustified situation. Thus, the following inequality must hold:

$$
\delta=\frac{a}{c} \sqrt{4 \pi \eta \rho_{0}}<\delta_{0}
$$

This inequality imposes certain restrictions on the acceptable values of the radius $a$ of the physical system and the invariant mass density $\rho_{0}$ in this system. For an example, we will estimate $\delta$ for the entire observable Universe, which at a scale of $100 \mathrm{Mpc}$ or more can be considered as a relativistic uniform system due to slight variation in the average mass density at different points.

From observations it follows that the Universe is very close to a state, in which spacetime is flat and almost Euclidean. In this case, according to the Lambda-CDM model the total mass-energy density of the Universe is close to the critical value $\rho_{c} \approx 10^{-26} \mathrm{~kg} / \mathrm{m}^{3}$ [20]. The size of the Universe can be estimated as the Hubble length $R_{H}=\frac{c}{H_{0}} \approx 10^{26} \mathrm{~m}$, where $H_{0}$ is the Hubble parameter. Taking $\rho_{c}$ in place of $\rho_{0}$, and also $R_{H}$ in place of $a$ and using the approximate equality $\eta \approx \frac{3}{5} G$ according to [5], we find the value $\delta_{U}=\frac{R_{H}}{c} \sqrt{4 \pi \eta \rho_{c}} \approx 1.7>\frac{\pi}{2}$ radians.

Since the angle $\delta_{U}$ is sufficiently large, for modeling the gravitational field of the Universe it is necessary to use the refined formulas with sines and cosines. As can be seen from the formula for the gravitational field strength inside the system (6), as the current radius $r$ increases to the value $R_{H}$ or more, the values $\delta_{r}=\frac{r}{c} \sqrt{4 \pi \eta \rho_{0}}, A_{r}=\sin \delta_{r}-\delta_{r} \cos \delta_{r}$ and the magnitude of the field strength $\Gamma_{i}$ first increase, but then begin to decrease. Under the boundary condition (16), that is at $\delta_{0} \approx 4.494$ radians, both $A_{r}$, and $\Gamma_{i}$, vanish, and at the boundary of the Universe, at $r>R_{H}$, gravitation tends to zero . 
On the other hand, the large-scale structure of the Universe is an observational fact, when the matter of clusters of galaxies near the boundary of the Universe does not form large spherical systems, but forms a network structure of clusters of galaxies. This can be explained as follows: at large distances gravitation seems to lose its strength, and the distant parts of the Universe have weaker influence, as compared to the closer parts, than they should by the Newton's law. We suppose that this effect is exactly described by the formula for the gravitational field strength inside the system (6).

But why should gravitation weaken at large distances? There is a model that combines the infinite nesting of matter and the Le Sage's theory of gravitation, and explains the weakening of gravitation by scattering of gravitons on the particles of space environment [21]. In this case, it turns out that the observable Universe in its scale is close enough to the limiting sizes of a system of stars and galaxies that can be kept from disruption by the ordinary gravitation force.

\section{Strong Gravitation at the Level of Nucleons}

Let us now pass on from large sizes and low mass density to the case of small sizes and high mass density, and again we will use (6) for the gravitational field strength, but already inside the proton. The proton fits well into the relativistic uniform model, since its average density of the order of $\rho_{p}=6 \times 10^{17} \mathrm{~kg} / \mathrm{m}^{3}$ at the proton radius $r_{p}=8.73 \times 10^{-16} \mathrm{~m}$, according to [22], is only 1.5 times less than the mass density in the center. As the gravitational constant at the atomic level we will use the strong gravitational constant $G_{s}=1.514 \times 10^{29} \mathrm{~m}^{3} /(\mathrm{kg} \cdot \mathrm{s})$, assuming that it is the strong gravitation that holds together the matter of nucleons and other particles [12].

Just as in (16), we will estimate the value $\delta$ for the proton using the approximate equality $\eta \approx \frac{3}{5} G_{s}:$

$$
\delta_{p}=\frac{r_{p}}{c} \sqrt{4 \pi \eta \rho_{p}} \approx 2.4<\delta_{0}
$$

As we can see, for the proton the angle $\delta_{p}$ is close enough to the limiting angle $\delta_{0}$. Hence it also follows that it is necessary to use sines and cosines in (6) and (7) in order to describe the gravitational field of strong gravitation inside and outside the proton.

Suppose now that we can add matter inside the proton volume, increasing the proton's mass density to a certain value $\rho_{0 m}$ in such a way that the angle $\delta_{p}$ becomes equal to the limiting angle $\delta_{0}$. This corresponds to the condition $\frac{r_{p}}{c} \sqrt{4 \pi \eta \rho_{0 m}}=\delta_{0} \approx 4.494$ radians, from which we obtain the estimate of the limiting invariant mass density $\rho_{0 m}=2.1 \times 10^{18} \mathrm{~kg} / \mathrm{m}^{3}$ and the estimate of the corresponding invariant energy density $\varepsilon_{0 m}=\rho_{0 m} c^{2}=1.9 \times 10^{35} \mathrm{~J} / \mathrm{m}^{3}$. In order to turn from these invariant values to the observed values, in the first approximation it suffices to multiply the invariant values by the Lorentz factor $\gamma_{c}$ of the motion of matter in the center of the proton. As is indicated in [1], this is equivalent to the fact that instead of mass $m$ we use the mass $m_{b}$, determined in (4), which is equal to the gravitational mass $m_{g}$ of the system.

On the other hand, in [23, 24] in the Le Sage's model of gravitation, the energy density of gravitons in the vacuum field was found, which was equal to $\varepsilon_{c}=7.4 \times 10^{35} \mathrm{~J} / \mathrm{m}^{3}$. From the equality $\varepsilon_{c} \approx \varepsilon_{0 m} \gamma_{c}$ we obtain the estimate of the Lorentz factor in the center of the proton: $\gamma_{c} \approx 1.9$. This also follows directly from (4), where we replace $m_{b}$ with the proton mass, replace $\frac{a}{c} \sqrt{4 \pi \eta \rho_{0}}$ 
with $\delta_{p}=\frac{r_{p}}{c} \sqrt{4 \pi \eta \rho_{p}} \approx 2.4$ radians, replace $a$ with the proton radius $r_{p}$, and take into account the ratio $\eta \approx \frac{3}{5} G_{s}$.

But why do we use equality $\varepsilon_{c} \approx \varepsilon_{0 m} \gamma_{c}$ ? The point is that by adding matter inside the proton up to the limiting invariant mass density $\rho_{0 m}=2.1 \times 10^{18} \mathrm{~kg} / \mathrm{m}^{3}$, according to (6) and (7) we decrease the gravitational field on the proton's surface to zero. In order to explain this, we need to refer to the Le Sage's model of gravitation. In this model, for the emergence of the gravitation force the gradient of the gravitons' flux is required that falls on the test body. As a rule, such a gradient arises between two bodies, leading to the gravitation force, that is, to the attraction of bodies. However, the gradient of the gravitons' flux on the proton's surface will disappear if the energy density $\varepsilon_{c}$ of the gravitons in the vacuum field becomes equal to the maximum energy density inside the proton, equal to $\varepsilon_{0 m} \gamma_{c}$. The proton will emit as many gravitons as the surrounding vacuum, and will become indistinguishable from the vacuum with respect to the energy density. In the absence of the gravitons' gradient the gravitational force disappears, which is reflected in expression (6) for the field strength in the limiting case at $\frac{r_{p}}{c} \sqrt{4 \pi \eta \rho_{0 m}}=\delta_{0}$.

Let us consider another situation involving strong gravitation. In particular, we will estimate the maximum radius $r_{G}$ of the system, which is held by strong gravitation and has the mass density equal to the critical density $\rho_{c} \approx 10^{-26} \mathrm{~kg} / \mathrm{m}^{3}$ in the observable Universe. For this, similarly to (16), the following condition must be fulfilled: $\frac{r_{G}}{c} \sqrt{4 \pi \eta \rho_{c}}<\delta_{0}$. Substituting here the approximate equality $\eta \approx \frac{3}{5} G_{s}$, we find $r_{G}<1.3 \times 10^{7} \mathrm{~m}$. As in the previous section for the case of the Universe, we can now assume that the strong gravitation stops acting at distances more than $r_{G}$ at the matter density $\rho_{c}$.

Dividing the Hubble length $R_{H}=\frac{c}{H_{0}} \approx 10^{26} \mathrm{~m}$ by $r_{G}$, we will obtain the relation $\frac{R_{H}}{r_{G}}>7.7 \times 10^{18}$. Next, we will use the coefficient $P$ of similarity in sizes between the atomic and stellar matter levels, for the proton and its analogue at the stellar level in the form of a neutron star. Dividing the radius of a typical neutron star with the mass of 1.35 Solar masses, which is approximately equal to $12 \mathrm{~km}$, by the radius of the proton, we will obtain $P=1.4 \times 10^{19}$. The value $P$ is of the same order as the ratio $\frac{R_{H}}{r_{G}}$. This means that the object with the radius $r_{G}$, held in equilibrium by the strong gravitation and consisting of nucleons or hydrogen atoms, is similar to the observable Universe, held in equilibrium by the ordinary gravitation and consisting of stars and their clusters.

In the general case, if in the condition $\frac{a}{c} \sqrt{4 \pi \eta \rho_{0}}<\delta_{0}$ the mass density $\rho_{0}$ is increased, then consequently the limiting size $a$ of the object decreases, for which the gravitational field strength is calculated in (6), for both the strong and ordinary gravitation. At sizes exceeding $r_{G}$, the strong gravitation ceases to act in the cosmological space. The ordinary gravitation is much weaker than the strong gravitation, but the range of action of the ordinary gravitation in cosmological space is much greater. Thus, the strong gravitation at the sizes exceeding $r_{G}$ does not fully disappear - the ordinary gravitation remains and acts instead of it. However, the action of the ordinary gravitation is 
also limited, at approximately $r>R_{H}=\frac{c}{H_{0}} \approx 10^{26} \mathrm{~m}$ it starts to decrease, as was shown in the previous section. Accordingly, at such large sizes the ordinary gravitation should be replaced by some other weaker gravitation with a larger radius of action.

\section{The Neutron Star}

In the previous section the estimate of the Lorentz factor at the center of the proton was obtained: $\gamma_{c} \approx 1.9$. Similarly, it is possible to calculate the Lorentz factor for the particles at the center of a typical neutron star with the mass of 1.35 Solar masses, the radius of $R_{s}=12 \mathrm{~km}$, and the average density of $\rho_{s}=3.7 \times 10^{17} \mathrm{~kg} / \mathrm{m}^{3}$. For the star we need to take into account the ratio $\eta \approx \frac{3}{5} G$ and the angle of $\delta_{s}=\frac{R_{s}}{c} \sqrt{4 \pi \eta \rho_{s}} \approx 0.546$ radians. Substituting now in (4) the star mass instead of $m_{b}, \delta_{s}$ instead of $\frac{a}{c} \sqrt{4 \pi \eta \rho_{0}}, \gamma_{c s}$ instead of $\gamma_{c}$, and replacing $a$ by the star radius $R_{s}$, we find $\gamma_{c s} \approx 1.04$. This allows us to calculate the kinetic energy $E_{k}=\left(\gamma_{c s}-1\right) m_{p} c^{2}$ of the proton as a certain typical particle, moving at the center of the neutron star, and to estimate the temperature at the center of the star: $T_{s} \approx 2.8 \times 10^{11} \mathrm{~K}$ using the equation $E_{k}=\frac{3}{2} k T_{s}$, where $k$ is the Boltzmann constant and $m_{p}$ is the proton mass. Previously, this temperature was calculated in [5] using the assumed, based on the calculations of other authors, changing of the mass density inside the star, where the following was obtained: $T_{s} \approx 5 \times 10^{11} \mathrm{~K}$. These values of the central temperatures can be considered the upper limit, which is valid for the moment of the star formation. In this short period of time the star is still in a hot state, the nucleons are moving randomly in its matter, and the approach described above is valid. Consequently, the Lorentz factor $\gamma_{c s} \approx 1.04$ for the particles at the center is the limiting value for the neutron star's matter. The same applies to the Lorentz factor $\gamma_{c} \approx 1.9$ at the center of the proton.

\section{Conclusions}

One of the conclusions in [25] was that the energy of the motionless matter of the uniform body in the form of a sphere in its proper static gravitational field is twice as large in its absolute value as the energy of the gravitational field itself. What will happen, if we turn into the relativistic uniform system, in which the matter particles are not motionless, but are moving with the Lorentz factor (2), depending on the current radius? The answer to this question follows from (13) - both in the classical and in the relativistic case, the relation between the energy of the particles in the field and the energy of the field itself remains unchanged.

In Section 4 we showed that, in the general case, the knowledge of the stress-energy tensors of fields is not enough to calculate the relativistic energy, and hence the corresponding inertial mass of the system. Thus, the covariant theory of gravitation differs significantly from the general theory of relativity, where it is possible to calculate the energy and mass by volume integration of the total stress-energy tensor of the system [26].

Applying the expressions for the potentials and the gravitational field strengths (1), (5), (6), (7) to the observable Universe and to the proton allows us to specify certain characteristic features of these objects. It turns out that these objects are in states close to the maximum possible, which requires an accurate account of the effects of the relativistic uniform model with respect to the gravitational field. Two extreme cases are possible - the largest possible sizes of the object $a$ and the low mass density $\rho_{0}$ (the Universe), and the extremely large mass density with small sizes (the 
proton, the neutron star). In (16) the condition $\delta=\frac{a}{c} \sqrt{4 \pi \eta \rho_{0}}<\delta_{0} \approx 4.494$ radians is provided, to which the object's sizes and mass density must correspond, if this object is held in equilibrium by its proper gravitational field. This allows us to estimate the radius of action of the strong and ordinary gravitation in the cosmological space. With the help of (4) it is also possible to calculate the maximum possible Lorentz factor of the matter particles at the center of the neutron star and at the center of the proton.

Thus, the results obtained with respect to the dependencies of the gravitational field inside and outside the bodies are in good agreement with the conclusions of the Le Sage's theory of gravitation, with the infinite nesting of matter, with the strong gravitation at the level of nucleons and with the concept of the dynamic force vacuum field.

At the next stage, it is planned to relate the conclusions of the covariant theory of gravitation and the results of the Einstein- $\Lambda$ model, described, for example, in [27] and [28], with respect to the energy of particles of the physical system.

\section{References}

[1] S.G. Fedosin, Relativistic energy and mass in the weak field limit, Jordan Journal of Physics. 8(1) (2015) 1-16.

[2] S.G. Fedosin The integral energy-momentum 4-vector and analysis of 4/3 problem based on the pressure field and acceleration field, American Journal of Modern Physics. 3(4) (2014) 152-167.

[3] V.I. Denisov, A.A. Logunov, The inertial mass defined in the general theory of relativity has no physical meaning, Theoretical and Mathematical Physics. 51(2) (1982) 421-426.

[4] R.I. Khrapko, The Truth about the energy-momentum tensor and pseudotensor, Gravitation and Cosmology. 20(4) (2014) 264-273.

[5] S.G. Fedosin, Estimation of the physical parameters of planets and stars in the gravitational equilibrium model, Canadian Journal of Physics. 94(4) (2016) 370-379.

[6] S.G. Fedosin, The virial theorem and the kinetic energy of particles of a macroscopic system in the general field concept, Continuum Mechanics and Thermodynamics. 29(2) (2016) 361371 .

[7] S.G. Fedosin, Two components of the macroscopic general field, Reports in Advances of Physical Sciences. 1(2) (2017).

[8] S.G. Fedosin, The metric outside a fixed charged body in the covariant theory of gravitation, International Frontier Science Letters. 1 (2014) 41-46.

[9] S. Fedosin, The physical theories and infinite hierarchical nesting of matter, Volume 2, LAP LAMBERT Academic Publishing, 2015. ISBN 978-3-659-71511-2.

[10] S.G. Fedosin, The pioneer anomaly in covariant theory of gravitation, Canadian Journal of Physics. 93(11) (2015) 1335-1342.

[11] A. Einstein, Gibt es eine Gravitationswirkung die der elektrodynamischen Induktionswirkung analog ist? Vierteljahrsschrift für gerichtliche Medizin und öffentliches Sanitätswesen. 44 (1912) 37-40.

[12] S.G. Fedosin, Fizika i filosofiia podobiia: ot preonov do metagalaktik, Perm, Russia, 1999. ISBN 5-8131-0012-1.

[13] M. Sharif, Z. Yousaf, Role of adiabatic index on the evolution of spherical gravitational collapse in Palatini f(R) gravity, Astrophys. Space Sci. 355 (2015) 317-331. 
[14] Z. Yousaf, M. Zaeem ul Haq Bhatti, Cavity evolution and instability constraints of relativistic interiors, Eur. Phys. J. C. 76 (2016) 267.

[15] Z. Yousaf, M. Zaeem ul Haq Bhatti, U. Farwa, Stability of compact stars in $\alpha \mathrm{R}^{2}+\beta\left(\mathrm{R} \gamma \delta \mathrm{T}^{\gamma \delta}\right)$ gravity, Mon. Not. R. Astron. Soc. 464(4) (2017) 4509-4519.

[16] M. Zaeem ul Haq Bhatti, Z. Yousaf, S. Hanif, Role of $\mathrm{f}(\mathrm{T})$ gravity on the evolution of collapsing stellar model, Phys. Dark Universe. 16 (2017) 34-40.

[17] S.G. Fedosin, The procedure of finding the stress-energy tensor and equations of vector field of any form, Advanced Studies in Theoretical Physics. 8(18) (2014) 771-779.

[18] S.G. Fedosin About the cosmological constant, acceleration field, pressure field and energy, Jordan Journal of Physics. 9(1) (2016) 1-30.

[19] S.G. Fedosin, The principle of least action in covariant theory of gravitation, Hadronic Journal. 35(1) (2012) 35-70

[20] C. Patrignani et al. (Particle Data Group), The review of particle physics, Chin. Phys. C. 40(10) (2016) 100001.

[21] S.G. Fedosin, Cosmic red shift, microwave background, and new particles, Galilean Electrodynamics. 23(1) (2012) 3-13.

[22] S.G. Fedosin, The radius of the proton in the self-consistent model, Hadronic Journal. 35(4) (2012) 349-363.

[23] S.G. Fedosin, The graviton field as the source of mass and gravitational force in the modernized Le Sage's model, Physical Science International Journal. 8(4) (2015) 1-18.

[24] S.G. Fedosin, The charged component of the vacuum field as the source of electric force in the modernized Le Sage's model, Journal of Fundamental and Applied Sciences. 8(3) (2016) 971-1020.

[25] S.G. Fedosin, The Hamiltonian in covariant theory of gravitation, Advances in Natural Science. 5(4) (2012) 55-75.

[26] V.A. Fock, The theory of space, time and gravitation, Macmillan, 1964.

[27] Z. Yousaf, Spherical relativistic vacuum core models in a $\Lambda$-dominated era, Eur. Phys. J. Plus. 132(2) (2017) 71.

[28] Z. Yousaf, Stellar filaments with Minkowskian core in the Einstein- $\Lambda$ gravity, Eur. Phys. J. Plus. 132(6) (2017) 276. 